\title{
Updates on bone health in children with gastrointe- stinal diseases
}

\author{
Hye Ran Yang, MD, PhD ${ }^{1,2}$ \\ 'Department of Pediatrics, Seoul \\ National University Bundang Hospital, \\ Seongnam, Korea \\ 2Department of Pediatrics, Seoul \\ National University College of Medi- \\ cine, Seoul, Korea
}

Chronic gastrointestinal diseases such as inflammatory bowel disease, malabsorption syndromes (e.g., intestinal lymphangiectasia, celiac disease, congenital chloride diarrhea, cystic fibrosis), and postsubtotal gastrectomy state or short-bowel syndrome after extensive bowel resection are related to poor bone health in pediatric patients due to increased risks of low bone mineral density, osteoporosis, and fractures. The pathophysiology of abnormal bone health in pediatric gastrointestinal diseases may present from inflammation to malabsorption. In children with chronic gastrointestinal diseases at high risk of poor bone health, routine evaluation using dual-energy X-ray absorptiometry and appropriate prevention or treatment strategies are needed.

Keywords: Bone mineral density, Gastrointestinal, Inflammatory bowel disease, Malabsorption, Short-bowel syndrome, Child

\section{Introduction}

Gastrointestinal (GI) diseases such as inflammatory bowel disease (IBD), malabsorption syndromes including celiac disease, and postgastrectomy state in gastric cancer survivors are related to high rates of osteopenia and osteoporosis in adults. ${ }^{1,2}$ According to a recent report, osteoporosis was found in $32 \%-42 \%$ of postgastrectomy, $18 \%-42 \%$ of IBD, and $15 \%-22 \%$ of celiac disease patients. ${ }^{1,2)}$ Further, the risk of fracture was also relatively high in adult patients with these GI diseases. ${ }^{1,2)}$

Similarly, some chronic GI diseases including IBD; rare malabsorption syndromes such as cystic fibrosis, intestinal lymphangiectasia, congenital chloride diarrhea, and celiac disease; and postsubtotal gastrectomy state or short-bowel syndrome after extensive bowel resection have been linked with osteopenia and osteoporosis in pediatric patients. However, data on poor bone health status in pediatric GI diseases remain limited.

This review article sought to provide updates on bone health issues in pediatric GI diseases using the most recent evidence.

\section{Bone health in children with IBD}

\section{Prevalence of osteoporosis in IBD}

IBD is a chronic relapsing GI condition with 2 major subtypes: Crohn disease (CD) and ulcerative colitis (UC). CD can affect any part of the GI tract, while UC is typically confined to the colonic mucosa. Beyond the GI tract, IBD is a kind of systemic disease manifesting with extraintestinal symptoms and complications, including osteopenia and osteoporosis. ${ }^{3)}$

Paired with increased risks for developing bone and mineral metabolism abnormalities, the prevalence of osteoporosis in adult IBD was reported to be $18 \%-42 \%$, while the risk of developing fractures is increased in IBD patients by $40 \%-60 \%$ relative to among healthy 
controls. ${ }^{1,2)}$ According to a recent systematic review and metaanalysis in 2018, risks of low bone mineral density (BMD) and fracture were reported to increase in adults with IBD compared with healthy sex- and age-matched controls. ${ }^{4)}$ IBD patients showed a significant decrease in mean BMD and $z$-scores versus controls at all sites. ${ }^{4)}$ The global risk of fracture was also increased in IBD patients compared to healthy controls (Relative risk, 1.38; 95\% confidence interval [CI], 1.11-1.73; $P=0.005)$, while the fracture risk was significantly increased among IBD patients only for vertebral fractures (odds ratio, 2.26; 95\% CI, $1.04-4.90 ; P<0.001)^{4)}$

Bone health in pediatric IBD was also assessed in several studies, revealing a higher rate of low BMD in pediatric IBD patients, especially in children with $\mathrm{CD} .{ }^{5)}$ A higher rate of bone fracture in children with IBD was reported to follow corticosteroid treatment with a steroid dose $>$ than $7.5 \mathrm{mg}$ per day, a lifetime cumulative dose greater than $5 \mathrm{~g}$, and more than 12 months of lifetime exposure. ${ }^{6}$

\section{Pathogenesis and risk factors of low BMD in IBD}

The pathogenesis of bone abnormality in IBD patients is multifactorial, including the effects of chronic inflammation and medical therapies such as corticosteroids on bone metabolism, the consequences of poor nutritional status, lack of physical activity, and influence of environmental factors (Fig. 1). ${ }^{7,8)}$

First, the influence of inflammatory cytokines on bone homeostasis is the main pathophysiology of low BMD and fracture risk because of the cytokine-mediated nature of chronic inflammation in IBD. ${ }^{9)}$ Receptor activator of nuclear factor$\kappa \mathrm{B}$ ligand (RANKL) activates osteoclasts and stimulates bone resorption, while the osteoprotegerin (OPG) system blocks osteoclast formation. ${ }^{9)}$ As proinflammatory cytokines such as tumor necrosis factor alpha- $\alpha$, interleukin (IL)-1, IL-6, IL-7, and IL-17 increase the ratio of RANKL to OPG, bone resorption is promoted in IBD patients.

Second, intestinal malabsorption resulting from disease activity or from extensive intestinal resection in patients with IBD can bring about deficiencies in calcium and vitamin D.

Third, the use of long-term high doses of systemic glucocorticoids to control disease activity of IBD also affects bone homeostasis by increasing bone resorption. ${ }^{7,8)}$ Malnutrition, immobilization, low body mass index, and hypogonadism may also play a role as risk factors of bone loss in IBD patients. ${ }^{7,8)}$ In pediatric IBD patients, it may be difficult to achieve peak bone mass if the disease begins in early life. ${ }^{8)}$

\section{Screening and treatment practice for bone health in IBD}

Regarding the screening and treatment of osteoporosis in adult patients with IBD, a Swiss IBD cohort study of 877 patients reported that 253 of the 877 participants underwent dual-energy X-ray absorptiometry (DXA), which revealed osteoporosis in about $20 \%$ of the tested patients. ${ }^{10)}$ In practice, treatment rates for IBD patients with osteoporosis were suboptimal; success rates of only $55 \%$ for calcium and $65 \%$ for vitamin $\mathrm{D}$ were noted, ${ }^{10)}$ remaining low at $53 \%$ for calcium and $58 \%$ for vitamin D even in patients also on systemic steroid therapy. ${ }^{10)}$ Only $29 \%$ of IBD patients with osteoporosis in that study were treated with bisphosphonate. Overall, the screening and treatment of osteoporosis were not timely in IBD patients, although appropriate calcium and vitamin D supplementation was significantly associated with improvement of BMD over time. $^{10)}$

In children with IBD, DXA can also be applied to screen bone health status. According to a recent study on musculoskeletal

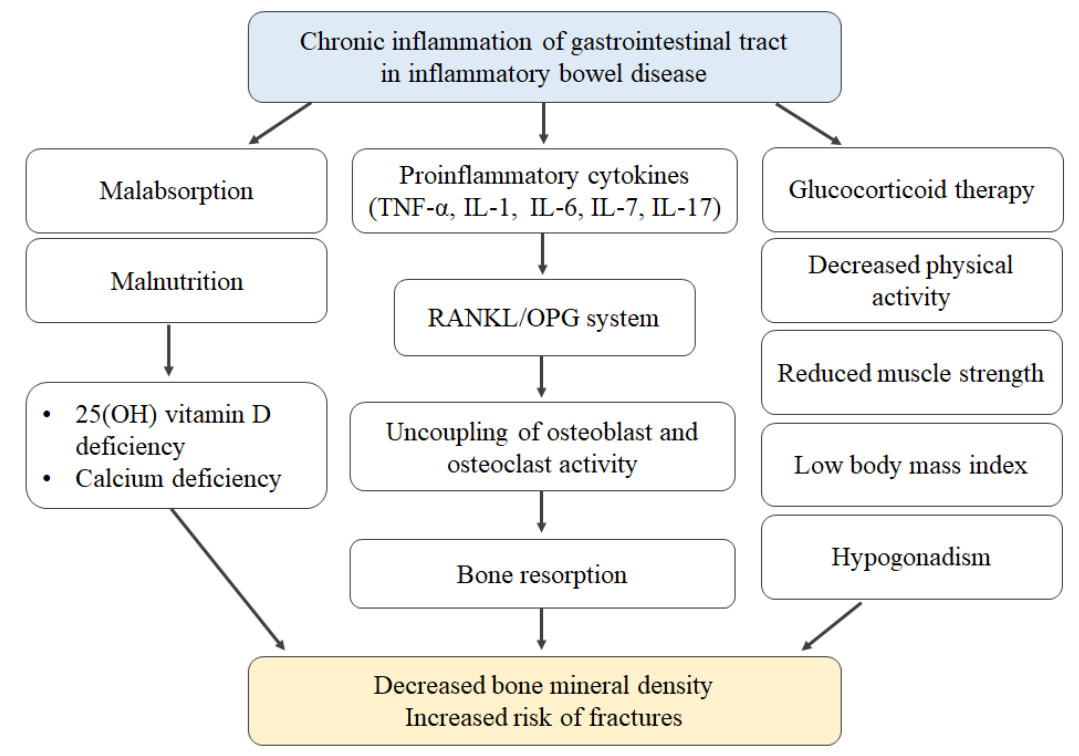

Fig. 1. Pathogenesis of abnormal bone health in pediatric patients with inflammatory bowel disease. TNF, tumor necrosis factor; IL, interleukin; RANKL, receptor activator of nuclear factor-KB ligand; OPG, osteoprotegerin. 
health in children with newly diagnosed CD, DXA, and peripheral quantitative computed tomography for elucidating bone and muscle mass and density were applied, and lumbar spine BMD and total body lean mass were both low in children with $\mathrm{CD}$, even though vertebral fracture was observed only in 1 patient. ${ }^{11)}$ Additionally, a recent study on pediatric-onset IBD focusing on risk of low BMD in adulthood over time reported that osteopenia and osteoporosis were highly prevalent in young IBD patients, and low BMD $z$-scores in adulthood positively correlated with low weight $z$-scores at the time of diagnosis in childhood. ${ }^{12)}$ Diminished peak bone mass in early adulthood resulted from pediatric-onset IBD and may increase the fracture risk during late adulthood.

Therefore, early interventions in pediatric IBD patients with low BMD are recommended for better bone health outcomes. All pediatric patients with IBD, especially $\mathrm{CD}$, using steroids; having a persistently active disease status; or showing risk factors such as poor nutrition, low calcium intake, limited physical activity, previous fracture history or family history of osteoporotic fracture, late menarche after 15 years of age; or smoking or excessive alcohol intake should be routinely screened for bone health status by means of DXA in addition to full correction of any potential calcium and vitamin D deficiencies to facilitate timely therapeutic intervention of patients at risk. ${ }^{8,10)}$

According to recommendations from the position paper by the Porto IBD group of the European Society of Pediatric Gastroenterology Hepatology and Nutrition (ESPGHAN), calcium intake should be monitored in children and adolescents with IBD, and low calcium intake should be supplemented. ${ }^{13)}$ ESPGHAN also recommended pursuing monitoring of vitamin $D$ level in all children with IBD and vitamin D supplementation in children with IBD when vitamin D deficiency is present, i.e., 25(OH) vitamin D concentration below $50 \mathrm{nmol} / \mathrm{L}$ or $20 \mathrm{ng} /$ $\mathrm{mL} .{ }^{13)}$

As for the efficacy and safety of medical treatment addressing low BMD in patients with IBD including calcium and vitamin D supplementation, bisphosphonate, fluoride, calcitonin, and lowimpact exercise, a recent meta-analysis and systematic review of 19 randomized controlled studies showed that bisphosphonate was effective and safe for treatment of low BMD and reduced the risk of vertebral fracture in IBD patients, while the evidence for other treatment options was insufficient to support their efficacy. ${ }^{14)}$

\section{Bone health in children with malabsorption syndromes}

Malabsorption syndromes such as primary intestinal lymphangiectasia (PIL), celiac disease, congenital chloride diarrhea, and cystic fibrosis are rare in children., ${ }^{1,2}$

PIL is a common etiology of protein-losing enteropathy characterized by abnormally dilated lymphatic ducts in the intestinal wall. ${ }^{15)}$ Because PIL affects the absorption of lipidsoluble vitamins, electrolytes, and minerals, malnutrition (vitamin D and calcium deficiency) related to osteomalacia and osteoporosis can develop in children with PIL. ${ }^{15)}$ There have been several case reports of low BMD with increased serum levels of parathyroid hormone and vitamin D in PIL, all of which were efficaciously treated with adequate calcium and vitamin D supplementation. ${ }^{15,16)}$

Celiac disease, i.e., gluten-sensitive enteropathy, is an immune-mediated systemic disorder elicited by gluten and related prolamines in genetically susceptible individuals and characterized by the presence of a variable combination of gluten-dependent clinical manifestations; celiac disease-specific antibodies such as anti-transglutaminase 2, antiendomysial antibody, gliadin, HLA-DQ2, or DQ8 haplotypes; and enteropathy with symptoms and signs of malabsorption. ${ }^{17)}$

Osteoporosis with hypocalcemia and hypocalciuria occurs as one of the extraintestinal manifestations in children with celiac disease. ${ }^{17)}$ To date, some severe cases of spontaneous bone fracture have been reported. Poor bone health in celiac disease is related to chronic malabsorption of calcium and vitamin $\mathrm{D}$.

In the screening and prevention of abnormal bone health, a recent guideline by the British Society of Gastroenterology recommended measurement of calcium, alkaline phosphatase, and vitamin D level in serum, with parathyroid hormone and calcium intake maintained at or above $1,000 \mathrm{mg} /$ day. ${ }^{17)}$ This guideline also supported continued measurement of BMD by DXA at intervals of 2 years in patients with ongoing villous atrophy, poor dietary adherence, and low BMD. ${ }^{17}$ Elsewhere, a recent position paper recommended adult patients with celiac disease undergo serum calcium, albumin, 25(OH) vitamin D parathyroid hormone, and 24-hour urine calcium testing at the time of diagnosis as well as DXA imaging in those at risk for osteoporosis, which should be repeated one to 2 years after initiation of a gluten-free diet. ${ }^{18)}$ Another guideline by the American Gastroenterology Association recommended that DXA scan be performed at one year after initiating a gluten-free diet in adults with celiac disease. ${ }^{19)}$

A study on bone health status in 74 children with celiac disease revealed that $16 \%$ of newly diagnosed celiac disease cases had BMD $z$-score less than -2, although there were no differences in BMD $z$-score between symptomatic and asymptomatic children. ${ }^{20)}$ In addition, age at diagnosis was inversely correlated with BMD $z$-score, and delayed diagnosis in children with celiac disease increased the risk of osteoporosis. ${ }^{20)}$ In pediatric patients with celiac disease, low BMD can be reversed by adherence to a gluten-free diet with restoration of normal peak BMD. Therefore, early routine evaluation for BMD using DXA and early nutritional intervention are recommended in children with celiac disease to assure better outcomes. A recent position paper recommended DXA scanning one year after diagnosis if gluten-free diet adherence is not strict. ${ }^{18)}$

\section{Bone health in children with short-bowel syndrome}

Short-bowel syndrome is an intestinal dysfunction, and 
complications following extensive loss of the small bowel can result in insufficient nutrient absorption and requirement for parenteral nutritional support. In clinical practice, extensive resection of the intestine in cases of necrotizing enterocolitis, intestinal atresia, volvulus, or gastroschisis is a primary cause of short-bowel syndrome. In children with short-bowel syndrome, prognosis factors include the length of the remnant bowel, bowel condition, preservation of the ileocecal valve, and stomy formation. ${ }^{21)}$ Usually, a total small-bowel length less than 40 $\mathrm{cm}$ necessitates more than 2 years of adaptation by the remnant bowel.

Malabsorption after extensive bowel resection results in fluid and electrolyte abnormalities and malnutrition in children. Metabolic changes due to loss of colonic regulation of gastric and small-bowel function lead to depletion of calcium, magnesium, and vitamin $\mathrm{D}$, resulting in demineralization of bone and low BMD. ${ }^{21)}$ Parenteral nutrition, persistent inflammation, steroid use, chronic metabolic acidosis, and renal insufficiency are additional factors that contribute to development of bone disease in patients with short-bowel syndrome. $^{21)}$

According to a retrospective study of 36 pediatric intestinal failure patients on parenteral nutrition for more than 30 days, BMD $z$-score less than -1.0 on DXA scanning was noted in $50 \%(\mathrm{n}=18)$ of the patients, serum $25(\mathrm{OH})$ vitamin D level less than $30 \mathrm{ng} / \mathrm{mL}$ was found in $63.8 \%$, and pathologic fracture was observed in $11.1 \%(n=4) .{ }^{22)}$ In this pediatric study, duration of parenteral nutrition was significantly associated with low BMD. $^{22)}$ In another retrospective study of 123 intestinal failure patients both children and young adults on total parenteral nutrition for more than 30 days, BMD $z$-score less than -2.0 was noted in $12.5 \%(\mathrm{n}=18)$, and serum $25(\mathrm{OH})$ vitamin D level less than $20 \mathrm{ng} / \mathrm{mL}$ was recorded in $39.8 \%(\mathrm{n}=49)$. In this pediatric study, duration of parenteral nutrition was significantly associated with low BMD. ${ }^{23)}$

Therefore, appropriate strategies for monitoring and preventing abnormal bone health are needed to secure better outcomes in children with short-bowel syndrome and intestinal failure receiving long-term parenteral nutrition.

\section{Conclusion}

Examples of abnormal bone health, including low BMD, osteopenia or osteoporosis, and fracture, are common complications of pediatric GI diseases. The pathophysiology of bone abnormality may be different according to disease entity, ranging from chronic inflammation to malabsorption and malnutrition. Regardless of underlying conditions, routine evaluation of bone health status using DXA and appropriate prevention or treatment strategies are needed in children with chronic GI diseases at high risk, especially in those with IBD, malabsorption syndrome, or short-bowel syndrome.

\section{Conflicts of interest}

No potential conflict of interest relevant to this article was reported.

\section{References}

1. Oh HJ, Ryu KH, Park BJ, Yoon BH. Osteoporosis and osteoporotic fractures in gastrointestinal disease. J Bone Metab 2018;25:213-7.

2. Katz S, Weinerman S. Osteoporosis and gastrointestinal disease. Gastroenterol Hepatol (N Y) 2010;6:506-17.

3. Sohn J, Chang EJ, Yang HR. Vitamin D status and bone mineral density in children with inflammatory bowel disease compared to those with functional abdominal pain. J Korean Med Sci 2017;32:961-7.

4. Szafors P, Che H, Barnetche T, Morel J, Gaujoux-Viala C, Combe B, et al. Risk of fracture and low bone mineral density in adults with inflammatory bowel diseases. A systematic literature review with meta-analysis. Osteoporos Int 2018;29:2389-97.

5. Sylvester FA, Wyzga N, Hyams JS, Davis PM, Lerer T, Vance $K$, et al. Natural history of bone metabolism and bone mineral density in children with inflammatory bowel disease. Inflamm Bowel Dis 2007;13:42-50.

6. Scott EM, Gaywood I, Scott BB. Guidelines for osteoporosis in coeliac disease and inflammatory bowel disease. British Society of Gastroenterology. Gut 2000;46 Suppl 1:i1-8.

7. Ghishan FK, Kiela PR. Advances in the understanding of mineral and bone metabolism in inflammatory bowel diseases. Am J Physiol Gastrointest Liver Physiol 2011;300:G191-201.

8. van Hogezand RA, Hamdy NA. Skeletal morbidity in inflammatory bowel disease. Scand J Gastroenterol Suppl 2006;(243):59-64.

9. Tilg H, Moschen AR, Kaser A, Pines A, Dotan I. Gut, inflammation and osteoporosis: basic and clinical concepts. Gut 2008;57:684-94.

10. Schüle S, Rossel JB, Frey D, Biedermann L, Scharl M, Zeitz J, et al. Widely differing screening and treatment practice for osteoporosis in patients with inflammatory bowel diseases in the Swiss IBD cohort study. Medicine (Baltimore) 2017;96:e6788.

11. Ward LM, Ma J, Rauch F, Benchimol EI, Hay J, Leonard MB, et al. Musculoskeletal health in newly diagnosed children with Crohn's disease. Osteoporos Int 2017;28:3169-77.

12. Guz-Mark A, Rinawi F, Egotubov O, Shimon I, Shamir R, Assa A. Pediatric-onset inflammatory bowel disease poses risk for low bone mineral density at early adulthood. Dig Liver Dis 2017;49:639-42.

13. Miele E, Shamir R, Aloi M, Assa A, Braegger C, Bronsky J, et al. Nutrition in Pediatric inflammatory bowel disease: a position paper on behalf of the porto inflammatory bowel disease group of the european society of pediatric gastroenterology, hepatology and nutrition. J Pediatr 
Gastroenterol Nutr 2018;66:687-708.

14. Melek J, Sakuraba A. Efficacy and safety of medical therapy for low bone mineral density in patients with inflammatory bowel disease: a meta-analysis and systematic review. Clin Gastroenterol Hepatol 2014;12:32-44.

15. Li XP, Shen WB, Long MQ, Meng XW, Lian XL, Yu M. Osteomalacia and osteoporosis associated with primary intestinal lymphangiectasis. Chin Med J (Engl) 2012;125:1836-8.

16. Sahli H, Ben Mbarek R, Elleuch M, Azzouz D, Meddeb N, Chéour E, et al. Osteomalacia in a patient with primary intestinal lymphangiectasis (Waldmann's disease). Joint Bone Spine 2008;75:73-5.

17. Ludvigsson JF, Bai JC, Biagi F, Card TR, Ciacci C, Ciclitira PJ, et al. Diagnosis and management of adult coeliac disease: guidelines from the British Society of Gastroenterology. Gut 2014;63:1210-28.

18. Fouda MA, Khan AA, Sultan MS, Rios LP, McAssey K, Armstrong D. Evaluation and management of skeletal health in celiac disease: position statement. Can J Gastroenterol 2012;26:819-29.

19. Bernstein CN, Leslie WD, Leboff MS. AGA technical review on osteoporosis in gastrointestinal diseases. Gastroenterology 2003;124:795-841.

20. Turner J, Pellerin G, Mager D. Prevalence of metabolic bone disease in children with celiac disease is independent of symptoms at diagnosis. J Pediatr Gastroenterol Nutr 2009;49:589-93.

21. Johnson E, Vu L, Matarese LE. Bacteria, bones, and stones: managing complications of short bowel syndrome. Nutr Clin Pract 2018;33:454-66.

22. Demehri FR, Simha S, Stephens L, Harris MB, Arnold MA, Brown PI, et al. Pediatric intestinal failure: predictors of metabolic bone disease. J Pediatr Surg 2015;50:958-62.

23. Ubesie AC, Heubi JE, Kocoshis SA, Henderson CJ, Mezoff AG, Rao MB, et al. Vitamin D deficiency and low bone mineral density in pediatric and young adult intestinal failure. J Pediatr Gastroenterol Nutr 2013;57:372-6. 